\title{
An approximation property of simple harmonic functions
}

Soon-Mo Jung*

${ }^{\text {"Correspondence: }}$ smjung@hongik.ac.kr

Mathematics Section, College of Science and Technology, Hongik

University, Jochiwon, 339-701, Korea

\begin{abstract}
In this paper, applying the power series method, we approximate analytic functions by simple harmonic functions in a neighborhood of zero.
\end{abstract}

\section{Introduction}

Differential equations have been studied for more than 300 years since the seventeenth century when the concepts of differentiation and integration were formulated by Newton and Leibniz. By use of differential equations, we can explain many natural phenomena: gravity, projectiles, wave, vibration, nuclear physics, and so on.

Let us consider a closed system which can be explained by the first-order linear differential equation, namely, $y^{\prime}(t)=\lambda y(t)$. The past, present, and future of this system are completely determined if we know the general solution and an initial condition of that differential equation. So, we can say that this system is 'predictable.' Sometimes, because of the disturbances (or noises) of the outside, the system may not be determined by $y^{\prime}(t)=\lambda y(t)$ but can only be explained by an inequality like $\left|y^{\prime}(t)-\lambda y(t)\right| \leq \varepsilon$. Then it is impossible to predict the exact future of the disturbed system.

Even though the system is not predictable exactly because of outside disturbances, we say the differential equation $y^{\prime}(t)=\lambda y(t)$ has the Hyers-Ulam stability if the 'real' future of the system follows the solution of $y^{\prime}(t)=\lambda y(t)$ with a bounded error. But if the error bound is 'too big,' we say that the differential equation $y^{\prime}(t)=\lambda y(t)$ does not have the Hyers-Ulam stability. Resonance is the case.

There is another way to explain the Hyers-Ulam stability. Usually the experiment (or the observed) data do not exactly coincide with theoretical expectations. We may express natural phenomena by use of equations, but because of the errors due to measurement or observance, the actual experiment data can almost always be a little bit off the expectations. If we used inequalities instead of equalities to explain natural phenomena, then these errors could be absorbed into the solutions of inequalities, i.e., those errors would no longer be errors. Considering this point of view, the Hyers-Ulam stability (of differential equations) is fundamental. (Hyers-Ulam stability is not same as the concept of the stability of differential equations which has been studied by many mathematicians for a long time.)

We will now introduce the concept of Hyers-Ulam stability of differential equations. Let $X$ be a normed space over a scalar field $\mathbb{K}$ and let $I \subset \mathbb{R}$ be an open interval, where $\mathbb{K}$ denotes either $\mathbb{R}$ or $\mathbb{C}$. Assume that $a_{0}, a_{1}, \ldots, a_{n}: I \rightarrow \mathbb{K}$ and $g: I \rightarrow X$ are continuous functions and that $y: I \rightarrow X$ is an $n$ times continuously differentiable function satisfying

(c) 2013 Jung; licensee Springer. This is an Open Access article distributed under the terms of the Creative Commons Attribution License (http://creativecommons.org/licenses/by/2.0), which permits unrestricted use, distribution, and reproduction in any medium, provided the original work is properly cited. 
the inequality

$$
\left\|a_{n}(x) y^{(n)}(x)+a_{n-1}(x) y^{(n-1)}(x)+\cdots+a_{1}(x) y^{\prime}(x)+a_{0}(x) y(x)+g(x)\right\| \leq \varepsilon
$$

for all $x \in I$ and for a given $\varepsilon>0$. If there exists an $n$ times continuously differentiable function $y_{0}: I \rightarrow X$ satisfying

$$
a_{n}(x) y_{0}^{(n)}(x)+a_{n-1}(x) y_{0}^{(n-1)}(x)+\cdots+a_{1}(x) y_{0}^{\prime}(x)+a_{0}(x) y_{0}(x)+g(x)=0
$$

and $\left\|y(x)-y_{0}(x)\right\| \leq K(\varepsilon)$ for all $x \in I$, where $K(\varepsilon)$ is an expression of $\varepsilon$ with $\lim _{\varepsilon \rightarrow 0} K(\varepsilon)=0$, then we say that the above differential equation has the Hyers-Ulam stability. For more detailed definitions of the Hyers-Ulam stability, we refer the reader to [1-6].

Hyers-Ulam stability of functional equations has been studied for more than 70 years. But the history of the Hyers-Ulam stability of differential equations is less than 20 years. For example, Obłoza seems to be the first author who investigated the Hyers-Ulam stability of linear differential equations in 1993 (see [7, 8]). Thereafter, Alsina and Ger published their paper [9], which handles the Hyers-Ulam stability of the linear differential equation $y^{\prime}(x)=y(x)$ : If a differentiable function $y(x)$ is a solution of the inequality $\left|y^{\prime}(x)-y(x)\right| \leq \varepsilon$ for any $x \in(a, \infty)$, then there exists a constant $c$ such that $\left|y(x)-c e^{x}\right| \leq 3 \varepsilon$ for all $x \in(a, \infty)$. We know that the general solution of the linear differential equation $y^{\prime}(x)=y(x)$ is $y(x)=c e^{x}$, where $c$ is a constant.

Therefore, we say that the differential equation $y^{\prime}(x)=y(x)$ has the Hyers-Ulam stability. If we can get a similar result with a control function $\varphi(x)$ in place of $\varepsilon$, we say that the differential equation $y^{\prime}(x)=y(x)$ has the Hyers-Ulam-Rassias stability.

In 2001 and 2002, Miura et al. [10] expanded Alsina and Ger's result by proving that the differential equation $y^{\prime}(x)=\lambda y(x)$ has the Hyers-Ulam stability. The author wrote a paper with Miura and Takahasi which expanded the result of Hyers-Ulam stability of that differential equation. To be more precise, we may choose a constant $c$ such that the solution of the inequality $\left|y^{\prime}(x)-\lambda y(x)\right| \leq \varphi(x)$ is not too far away from $c e^{\lambda x}$ in the sense of upper norm (see [11]).

Recently, the author applied the power series method to studying the Hyers-Ulam stability of several types of linear differential equations of second order (see [12-17]).

In Section 3 of this paper, we apply the power series method to prove the Hyers-Ulam stability of the simple harmonic oscillator equation

$$
y^{\prime \prime}(x)+\omega^{2} y(x)=0
$$

This paper is an extension and an improvement of the previous paper [16]. We denote by $\mathbb{N}_{0}$ the set of all nonnegative integers.

\section{Inhomogeneous simple harmonic oscillator equation}

Let $\omega$ be a positive constant. A function is called a simple harmonic oscillator function if it satisfies the simple harmonic oscillator equation (1.1), which plays an important role in quantum mechanics. 
Theorem 2.1 Let $L$ be a real number with $0 \leq L<1$. Assume that the radius of convergence of power series $\sum_{m=0}^{\infty} a_{m} x^{m}$ is $\rho_{1}>0$, and a sequence $\left\{c_{m}\right\}$ satisfies the recurrence relation

$$
(m+2)(m+1) c_{m+2}+\omega^{2} c_{m}=a_{m}
$$

for any $m \in \mathbb{N}_{0}$. If

$$
\lim _{m \rightarrow \infty}\left|\frac{a_{m-1}}{m a_{m}}\right|=\frac{L}{\omega},
$$

then every solution $y:\left(-\rho_{1}, \rho_{1}\right) \rightarrow \mathbb{C}$ of the inhomogeneous simple harmonic oscillator equation

$$
y^{\prime \prime}(x)+\omega^{2} y(x)=\sum_{m=0}^{\infty} a_{m} x^{m}
$$

can be expressed by

$$
y(x)=y_{h}(x)+\sum_{m=0}^{\infty} c_{m} x^{m}
$$

for all $x \in\left(-\rho_{1}, \rho_{1}\right)$, where $y_{h}(x)$ is a simple harmonic oscillator function and the value of $c_{m}$ is given by (2.4).

Proof We can use induction on $m$ and prove that if a sequence $\left\{c_{m}\right\}$ satisfies the relation (2.1), then

$$
\left\{\begin{array}{l}
c_{2 m}=\sum_{i=0}^{m-1}(-1)^{m-i-1} \frac{(2 i) !}{(2 m) !} \omega^{2 m-2 i-2} a_{2 i}+(-1)^{m} \frac{\omega^{2 m}}{(2 m) !} c_{0}, \\
c_{2 m+1}=\sum_{i=0}^{m-1}(-1)^{m-i-1} \frac{(2 i+1) !}{(2 m+1) !} \omega^{2 m-2 i-2} a_{2 i+1}+(-1)^{m} \frac{\omega^{2 m}}{(2 m+1) !} c_{1}
\end{array}\right.
$$

for all $m \in \mathbb{N}_{0}$. We omit the proof.

Due to (2.2), there exists an $m_{0} \in \mathbb{N}$ such that $a_{m} \neq 0$ for any integer $m \geq m_{0}$. Moreover, for any real number $\delta$ with $L<\delta<1$, we can choose an integer $m_{\delta}$ such that $m_{\delta}>m_{0}$ and

$$
\left|\frac{\omega a_{m-1}}{m a_{m}}\right| \leq \delta
$$

for all integers $m \geq m_{\delta}$. We now define a real constant $M>0$ by

$$
M=\max _{\substack{n \in\left\{1,2, \ldots, m_{\delta}-1\right\} \\ a_{n} \neq 0}} \max _{\substack{i \in\{0,1, \ldots, n-1\} \\ \omega^{i}}}\left|\frac{\omega^{n}}{\frac{i !}{n !}} \frac{a_{i}}{a_{n}}\right| .
$$

If $m>i \geq m_{\delta}-1$, it then follows from (2.5) that

$$
\left|\frac{\omega^{m}}{\omega^{i}} \frac{i !}{m !} \frac{a_{i}}{a_{m}}\right|=\prod_{k=i+1}^{m}\left|\frac{\omega a_{k-1}}{k a_{k}}\right| \leq \delta^{m-i} .
$$


If $m \geq m_{\delta}-1>i$, it then follows from (2.5) and (2.6) that

$$
\left|\frac{\omega^{m}}{\omega^{i}} \frac{i !}{m !} \frac{a_{i}}{a_{m}}\right|=\left|\frac{\omega^{m_{\delta}-1}}{\omega^{i}} \frac{i !}{\left(m_{\delta}-1\right) !} \frac{a_{i}}{a_{m_{\delta}-1}}\right| \prod_{k=m_{\delta}}^{m}\left|\frac{\omega a_{k-1}}{k a_{k}}\right| \leq M \delta^{m-m_{\delta}+1} .
$$

If $m_{\delta}-1>m>i$ and $a_{m} \neq 0$, then (2.6) implies that

$$
\left|\frac{\omega^{m}}{\omega^{i}} \frac{i !}{m !} \frac{a_{i}}{a_{m}}\right| \leq M
$$

In view of (2.4), (2.7), and (2.8), for any sufficiently large integer $m$, there exists a real constant $C>0$ such that

$$
\begin{aligned}
\left|c_{2 m}\right|= & \frac{\left|a_{2 m}\right|}{\omega^{2}}\left|\sum_{i=0}^{m-1}(-1)^{m-i-1} \frac{\omega^{2 m}}{\omega^{2 i}} \frac{(2 i) !}{(2 m) !} \frac{a_{2 i}}{a_{2 m}}+(-1)^{m} \frac{\omega^{m_{0}+2}}{m_{0} !} \frac{c_{0}}{a_{m_{0}}} \frac{\omega^{2 m}}{\omega^{m_{0}}} \frac{m_{0} !}{(2 m) !} \frac{a_{m_{0}}}{a_{2 m}}\right| \\
\leq & \frac{\left|a_{2 m}\right|}{\omega^{2}}\left[\sum_{0 \leq i<\left(m_{\delta}-1\right) / 2}\left|\frac{\omega^{2 m}}{\omega^{2 i}} \frac{(2 i) !}{(2 m) !} \frac{a_{2 i}}{a_{2 m}}\right|+\sum_{\left(m_{\delta}-1\right) / 2 \leq i<m}\left|\frac{\omega^{2 m}}{\omega^{2 i}} \frac{(2 i) !}{(2 m) !} \frac{a_{2 i}}{a_{2 m}}\right|\right. \\
& \left.+\frac{\omega^{m_{0}+2}}{m_{0} !}\left|\frac{c_{0}}{a_{m_{0}}}\right|\left|\frac{\omega^{2 m}}{\omega^{m_{0}}} \frac{m_{0} !}{(2 m) !} \frac{a_{m_{0}}}{a_{2 m}}\right|\right] \\
\leq & \frac{\left|a_{2 m}\right|}{\omega^{2}}\left[\frac{m_{\delta}+1}{2} M \delta^{2 m-m_{\delta}+1}+\sum_{\left(m_{\delta}-1\right) / 2 \leq i<m} \delta^{2 m-2 i}\right. \\
& \left.+\frac{\omega^{m_{0}+2}}{m_{0} !}\left|\frac{c_{0}}{a_{m_{0}}}\right| \max \{1, M\} \delta^{2 m-m_{\delta}+1}\right] \\
\leq & C\left|a_{2 m}\right| .
\end{aligned}
$$

Hence, it holds that

$$
\limsup _{m \rightarrow \infty}\left|c_{2 m}\right|^{1 /(2 m)} \leq \limsup _{m \rightarrow \infty} C^{1 /(2 m)}\left|a_{2 m}\right|^{1 /(2 m)}=\limsup _{m \rightarrow \infty}\left|a_{2 m}\right|^{1 /(2 m)} .
$$

Similarly, we have

$$
\limsup _{m \rightarrow \infty}\left|c_{2 m+1}\right|^{1 /(2 m+1)} \leq \limsup _{m \rightarrow \infty}\left|a_{2 m+1}\right|^{1 /(2 m+1)},
$$

which implies that the radius of convergence of power series $\sum_{m=0}^{\infty} c_{m} x^{m}$ is at least $\rho_{1}$. Moreover, we notice that the radius of convergence of a general solution of the simple harmonic oscillator equation (1.1) is $\rho_{0}=\infty$.

Since $x=0$ is an ordinary point, we can substitute $\sum_{m=0}^{\infty} c_{m} x^{m}$ for $y(x)$ and use the formal multiplication of power series and consider (2.1) to get

$$
\begin{aligned}
y^{\prime \prime}(x)+\omega^{2} y(x) & =\sum_{m=0}^{\infty}(m+2)(m+1) c_{m+2} x^{m}+\omega^{2} \sum_{m=0}^{\infty} c_{m} x^{m} \\
& =\sum_{m=0}^{\infty}\left[(m+2)(m+1) c_{m+2}+\omega^{2} c_{m}\right] x^{m} \\
& =\sum_{m=0}^{\infty} a_{m} x^{m}
\end{aligned}
$$


for all $x \in\left(-\rho_{1}, \rho_{1}\right)$. That is, $\sum_{m=0}^{\infty} c_{m} x^{m}$ is a particular solution of the inhomogeneous simple harmonic oscillator equation (2.3) and hence every solution $y:\left(-\rho_{1}, \rho_{1}\right) \rightarrow \mathbb{C}$ of (2.3) can be expressed by

$$
y(x)=y_{h}(x)+\sum_{m=0}^{\infty} c_{m} x^{m}
$$

where $y_{h}(x)$ is a simple harmonic oscillator function.

\section{Approximate simple harmonic oscillator equation}

Let $\rho_{1}$ be a positive constant. We denote by $\mathcal{C}$ the set of all functions $y:\left(-\rho_{1}, \rho_{1}\right) \rightarrow \mathbb{C}$ with the following properties:

(a) $y(x)$ is expressible by a power series $\sum_{m=0}^{\infty} b_{m} x^{m}$ whose radius of convergence is at least $\rho_{1}$;

(b) There exists a constant $K \geq 0$ such that $\sum_{m=0}^{\infty}\left|a_{m} x^{m}\right| \leq K\left|\sum_{m=0}^{\infty} a_{m} x^{m}\right|$ for any $x \in\left(-\rho_{1}, \rho_{1}\right)$, where $a_{m}=(m+2)(m+1) b_{m+2}+\omega^{2} b_{m} \neq 0$ for all $m \in \mathbb{N}_{0}$.

If we define

$$
\left(y_{1}+y_{2}\right)(t)=y_{1}(t)+y_{2}(t) \quad \text { and } \quad\left(\lambda y_{1}\right)(t)=\lambda y_{1}(t)
$$

for all $y_{1}, y_{2} \in \mathcal{C}$ and $\lambda \in \mathbb{C}$, then $\mathcal{C}$ is a vector space over complex numbers. We remark that the set $\mathcal{C}$ is large enough to be a vector space.

Now, we prove the main theorem of this paper.

Theorem 3.1 Let $L$ be a real number with $|L|<1$. Assume that $y:\left(-\rho_{1}, \rho_{1}\right) \rightarrow \mathbb{C}$ is an arbitrary function belonging to $\mathcal{C}$ whose power series coefficients satisfy either

$$
\begin{aligned}
& \lim _{m \rightarrow \infty} \frac{b_{m-1}}{m b_{m}}=\frac{L}{\omega} \quad \text { or } \quad \lim _{m \rightarrow \infty} \frac{b_{2 m-1}}{2 m b_{2 m}}=-\frac{L}{\omega} \text { and } \\
& \lim _{m \rightarrow \infty} \frac{b_{2 m}}{(2 m+1) b_{2 m+1}}=\frac{L}{\omega},
\end{aligned}
$$

where $\omega$ is a positive number. If $y$ satisfies the differential inequality

$$
\left|y^{\prime \prime}(x)+\omega^{2} y(x)\right| \leq \varepsilon
$$

for all $x \in\left(-\rho_{1}, \rho_{1}\right)$ and for some $\varepsilon>0$, then there exists a solution $y_{h}:\left(-\rho_{1}, \rho_{1}\right) \rightarrow \mathbb{C}$ of the simple harmonic oscillator equation (1.1) such that

$$
\left|y(x)-y_{h}(x)\right| \leq C K \varepsilon
$$

for all $x \in\left(-\rho_{1}, \rho_{1}\right)$, where $C$ is determined by (3.3) and $K$ is defined in (b).

Proof We will prove this theorem under the first condition of (3.1). Let $\rho_{2}$ be the radius of convergence of the power series $\sum_{m=0}^{\infty} c_{m} x^{m}$ which is a particular solution of the inhomogeneous simple harmonic oscillator equation (2.3), where $c_{m}$ is defined in (2.4). It follows 
from (b) and the first condition of (3.1) that

$$
\begin{aligned}
\lim _{m \rightarrow \infty}\left|\frac{a_{m-1}}{a_{m}}\right| & =\lim _{m \rightarrow \infty}\left|\frac{(m+1) m b_{m+1}+\omega^{2} b_{m-1}}{(m+2)(m+1) b_{m+2}+\omega^{2} b_{m}}\right| \\
& =\lim _{m \rightarrow \infty}\left|\frac{(m+1) m b_{m+1}}{(m+2)(m+1) b_{m+2}}\right|\left|\frac{1+\frac{\omega b_{m-1}}{m b_{m}} \frac{\omega b_{m}}{(m+1) b_{m+1}}}{1+\frac{\omega b_{m}}{(m+1) b_{m+1}} \frac{\omega b_{m+1}}{(m+2) b_{m+2}}}\right| \\
& =\lim _{m \rightarrow \infty}\left|\frac{b_{m+1}}{b_{m+2}}\right|,
\end{aligned}
$$

i.e., it holds that $\lim _{m \rightarrow \infty}\left|a_{m-1} / m a_{m}\right|=|L| / \omega$. In view of the proof of Theorem 2.1, it holds that $\rho_{2} \geq \rho_{1}$.

Similarly as in the proof of Theorem 2.1, it follows from (2.4), (2.7), (2.8), (2.9), and (2.10) that there exists a real number $C>0$ such that

$$
\left|c_{m}\right| \leq C\left|a_{m}\right|
$$

for any $m \in \mathbb{N}_{0}$.

By the same argument presented in the proof of Theorem 2.1 with substitution of $\sum_{m=0}^{\infty} b_{m} x^{m}$ (instead of $\left.\sum_{m=0}^{\infty} c_{m} x^{m}\right)$ for $y(x)$, it follows from (b) that

$$
y^{\prime \prime}(x)+\omega^{2} y(x)=\sum_{m=0}^{\infty} a_{m} x^{m}
$$

for all $x \in\left(-\rho_{1}, \rho_{1}\right)$. In view of (b), there exists a constant $K \geq 0$ such that

$$
\sum_{m=0}^{\infty}\left|a_{m} x^{m}\right| \leq K\left|\sum_{m=0}^{\infty} a_{m} x^{m}\right|
$$

for all $x \in\left(-\rho_{1}, \rho_{1}\right)$.

Moreover, by using (3.2), (3.4), and (3.5), we get

$$
\sum_{m=0}^{\infty}\left|a_{m} x^{m}\right| \leq K\left|\sum_{m=0}^{\infty} a_{m} x^{m}\right| \leq K \varepsilon
$$

for any $x \in\left(-\rho_{1}, \rho_{1}\right)$. (That is, the radius of convergence of power series $\sum_{m=0}^{\infty} a_{m} x^{m}$ is at least $\rho_{1}$.)

Since $\left\{c_{m}\right\}$ satisfies the recurrence relation (2.1), according to Theorem 2.1 and (3.4), $y(x)$ can be written as

$$
y(x)=y_{h}(x)+\sum_{m=0}^{\infty} c_{m} x^{m}
$$

for all $x \in\left(-\rho_{1}, \rho_{1}\right)$, where $y_{h}(x)$ is a solution of the simple harmonic oscillator equation (1.1).

By the same argument as in the proof of Theorem 2.1 with substitution of $\sum_{m=0}^{\infty} c_{m} x^{m}$ for $y(x)$, since $\left\{c_{m}\right\}$ satisfies the relation (2.1), it is easy to see that $\sum_{m=0}^{\infty} c_{m} x^{m}$ is a particular 
solution of (3.4). Hence, it follows from (3.3), (3.6), and (3.7) that

$$
\left|y(x)-y_{h}(x)\right| \leq \sum_{m=0}^{\infty}\left|c_{m} x^{m}\right| \leq C \sum_{m=0}^{\infty}\left|a_{m} x^{m}\right| \leq C K \varepsilon
$$

for all $x \in\left(-\rho_{1}, \rho_{1}\right)$.

We remark that Theorem 3.1 is true whether the 'radius' of the domain interval of $y$ is larger than one or not, while [16, Theorem 3.1] holds only when the function $y$ has the domain whose 'radius' is not larger than one.

\section{An example}

Let $\varepsilon, \omega$, and $\omega_{0}$ be positive real numbers satisfying

$$
0<\omega_{0}^{2}-\omega^{2} \leq \frac{\varepsilon}{\sqrt{2}} \csc \left(\frac{\pi}{4}+\frac{1}{2}\right)
$$

Moreover, we set $\rho_{1}=1 /\left(2 \omega_{0}\right)$.

We will show that if $y(x)=\cos \omega_{0} x+\sin \omega_{0} x$ for all $x \in\left(-\rho_{1}, \rho_{1}\right)$, then $y \in \mathcal{C}$ with the constant $K=79 / 19$. We know that the sine function $y(x)=\cos \omega_{0} x+\sin \omega_{0} x$ can be expressed by the power series

$$
y(x)=\sum_{m=0}^{\infty} \frac{(-1)^{m} \omega_{0}^{2 m} x^{2 m}}{(2 m) !}+\sum_{m=0}^{\infty} \frac{(-1)^{m} \omega_{0}^{2 m+1} x^{2 m+1}}{(2 m+1) !}=\sum_{m=0}^{\infty} b_{m} x^{m}
$$

where we set

$$
b_{2 m}=\frac{(-1)^{m} \omega_{0}^{2 m}}{(2 m) !} \quad \text { and } \quad b_{2 m+1}=\frac{(-1)^{m} \omega_{0}^{2 m+1}}{(2 m+1) !}
$$

for every $m \in \mathbb{N}_{0}$. Hence, $y$ satisfies the condition (a).

If we define

$$
a_{2 m}=(2 m+2)(2 m+1) b_{2 m+2}+\omega^{2} b_{2 m}=\frac{(-1)^{m}\left(\omega^{2}-\omega_{0}^{2}\right) \omega_{0}^{2 m}}{(2 m) !}
$$

and

$$
a_{2 m+1}=(2 m+3)(2 m+2) b_{2 m+3}+\omega^{2} b_{2 m+1}=\frac{(-1)^{m}\left(\omega^{2}-\omega_{0}^{2}\right) \omega_{0}^{2 m+1}}{(2 m+1) !}
$$

for any $m \in \mathbb{N}_{0}$, then we have

$$
\begin{aligned}
\sum_{m=0}^{\infty}\left|a_{m} x^{m}\right| & =\sum_{m=0}^{\infty}\left(\left|a_{4 m} x^{4 m}\right|+\left|a_{4 m+1} x^{4 m+1}\right|+\left|a_{4 m+2} x^{4 m+2}\right|+\left|a_{4 m+3} x^{4 m+3}\right|\right) \\
& =\left|\omega^{2}-\omega_{0}^{2}\right| \sum_{m=0}^{\infty}\left(\frac{\left|\omega_{0} x\right|^{4 m}}{(4 m) !}+\frac{\left|\omega_{0} x\right|^{4 m+1}}{(4 m+1) !}+\frac{\left|\omega_{0} x\right|^{4 m+2}}{(4 m+2) !}+\frac{\left|\omega_{0} x\right|^{4 m+3}}{(4 m+3) !}\right) .
\end{aligned}
$$


Furthermore, by considering $\left|\omega_{0} x\right|<1 / 2$ and by a long and tedious calculation, we get

$$
\begin{aligned}
\left|\sum_{m=0}^{\infty} a_{m} x^{m}\right| & =\left|\sum_{m=0}^{\infty}\left(a_{4 m} x^{4 m}+a_{4 m+1} x^{4 m+1}+a_{4 m+2} x^{4 m+2}+a_{4 m+3} x^{4 m+3}\right)\right| \\
& =\left|\omega^{2}-\omega_{0}^{2}\right|\left|\sum_{m=0}^{\infty}\left(\frac{\left(\omega_{0} x\right)^{4 m}}{(4 m) !}+\frac{\left(\omega_{0} x\right)^{4 m+1}}{(4 m+1) !}-\frac{\left(\omega_{0} x\right)^{4 m+2}}{(4 m+2) !}-\frac{\left(\omega_{0} x\right)^{4 m+3}}{(4 m+3) !}\right)\right| \\
& \geq \frac{19}{79}\left|\omega^{2}-\omega_{0}^{2}\right| \sum_{m=0}^{\infty}\left(\frac{\left|\omega_{0} x\right|^{4 m}}{(4 m) !}+\frac{\left|\omega_{0} x\right|^{4 m+1}}{(4 m+1) !}+\frac{\left|\omega_{0} x\right|^{4 m+2}}{(4 m+2) !}+\frac{\left|\omega_{0} x\right|^{4 m+3}}{(4 m+3) !}\right) \\
& =\frac{19}{79} \sum_{m=0}^{\infty}\left|a_{m} x^{m}\right|
\end{aligned}
$$

for all $x \in\left(-\rho_{1}, \rho_{1}\right)$. Therefore, we conclude that

$$
\sum_{m=0}^{\infty}\left|a_{m} x^{m}\right| \leq \frac{79}{19}\left|\sum_{m=0}^{\infty} a_{m} x^{m}\right|
$$

for all $x \in\left(-\rho_{1}, \rho_{1}\right)$, i.e., (b) is satisfied with $K=79 / 19$ and $\rho_{1}=1 /\left(2 \omega_{0}\right)$, and hence $y \in \mathcal{C}$.

Now, we see that

$$
\lim _{m \rightarrow \infty} \frac{b_{2 m-1}}{2 m b_{2 m}}=-\frac{L}{\omega} \text { and } \lim _{m \rightarrow \infty} \frac{b_{2 m}}{(2 m+1) b_{2 m+1}}=\frac{L}{\omega},
$$

where $L=\omega / \omega_{0}<1$. Furthermore, it follows from (4.1) that

$$
\begin{aligned}
\left|y^{\prime \prime}(x)+\omega^{2} y(x)\right| & =\sqrt{2}\left|\omega^{2}-\omega_{0}^{2}\right|\left|\sin \left(\omega_{0} x+\frac{\pi}{4}\right)\right| \\
& \leq \sqrt{2}\left|\omega^{2}-\omega_{0}^{2}\right| \sin \left(\frac{\pi}{4}+\frac{1}{2}\right) \\
& \leq \varepsilon
\end{aligned}
$$

for all $x \in\left(-\rho_{1}, \rho_{1}\right)$.

We remark that we can take zeros as the values of $c_{0}$ and $c_{1}$ in (2.4) without changing the validity of Theorem 2.1. If we set $c_{0}=c_{1}=0, m_{0}=0$, and $m_{\delta}=1$, then it follows from (2.10) that

$$
\left|c_{2 m}\right| \leq \frac{\left|a_{2 m}\right|}{\omega^{2}} \sum_{i=0}^{m-1}\left|\frac{\omega^{2 m}}{\omega^{2 i}} \frac{(2 i) !}{(2 m) !} \frac{a_{2 i}}{a_{2 m}}\right| \leq \frac{\left|a_{2 m}\right|}{\omega^{2}} \sum_{i=0}^{m-1} \delta^{2 m-2 i} \leq \frac{\delta^{2}}{\omega^{2}\left(1-\delta^{2}\right)}\left|a_{2 m}\right|
$$

for any $\delta$ with $|L|<\delta<1$ and for all $m \in \mathbb{N}_{0}$. Similarly, we have

$$
\left|c_{2 m+1}\right| \leq \frac{\delta^{2}}{\omega^{2}\left(1-\delta^{2}\right)}\left|a_{2 m+1}\right|
$$

for all $\delta$ satisfying $|L|<\delta<1$ and for all $m \in \mathbb{N}_{0}$. Hence, we get

$$
\left|c_{m}\right| \leq \frac{\delta^{2}}{\omega^{2}\left(1-\delta^{2}\right)}\left|a_{m}\right|
$$


for all $\delta$ satisfying $|L|<\delta<1$ and for all $m \in \mathbb{N}_{0}$, i.e., it holds that

$$
\left|c_{m}\right| \leq \lim _{\delta \rightarrow|L|} \frac{\delta^{2}}{\omega^{2}\left(1-\delta^{2}\right)}\left|a_{m}\right|=\frac{1}{\omega_{0}^{2}-\omega^{2}}\left|a_{m}\right|
$$

for all $m \in \mathbb{N}_{0}$.

According to Theorem 3.1, there exists a solution $y_{h}:\left(-\rho_{1}, \rho_{1}\right) \rightarrow \mathbb{C}$ of the homogeneous differential equation (1.1) such that

$$
\left|\cos \omega_{0} x+\sin \omega_{0} x-y_{h}(x)\right| \leq \frac{79}{19} \frac{\varepsilon}{\omega_{0}^{2}-\omega^{2}}
$$

for all $x \in\left(-1 /\left(2 \omega_{0}\right), 1 /\left(2 \omega_{0}\right)\right)$.

\section{Competing interests}

The author declares that he has no competing interests.

\section{Authors' contributions}

The author declares that this paper is his original paper.

\section{Acknowledgements}

This research was supported by the Basic Science Research Program through the National Research Foundation of Korea (NRF) funded by the Ministry of Education, Science and Technology (No. 2011-0004919).

\section{Received: 5 September 2012 Accepted: 16 December 2012 Published: 3 January 2013}

\section{References}

1. Czerwik, S: Functional Equations and Inequalities in Several Variables. World Scientific, River Edge (2002)

2. Hyers, DH: On the stability of the linear functional equation. Proc. Natl. Acad. Sci. USA 27, 222-224 (1941)

3. Hyers, DH, Isac, G, Rassias, TM: Stability of Functional Equations in Several Variables. Birkhäuser, Boston (1998)

4. Jung, S-M: Hyers-Ulam-Rassias Stability of Functional Equations in Nonlinear Analysis. Springer Optimization and Its Applications, vol. 48. Springer, New York (2011)

5. Rassias, TM: On the stability of the linear mapping in Banach spaces. Proc. Am. Math. Soc. 72, 297-300 (1978)

6. Ulam, SM: Problems in Modern Mathematics. Wiley, New York (1964)

7. Obłoza, M: Hyers stability of the linear differential equation. Rocz. Nauk.-Dydakt. - Wyż. Szk. Pedagog. Im. Komis. Eduk. Nar. Pr. Mat. 13, 259-270 (1993)

8. Obłoza, M: Connections between Hyers and Lyapunov stability of the ordinary differential equations. Rocz. Nauk.-Dydakt. - Wyż. Szk. Pedagog. Im. Komis. Eduk. Nar., Pr. Mat. 14, 141-146 (1997)

9. Alsina, C, Ger, R: On some inequalities and stability results related to the exponential function. J. Inequal. Appl. 2, 373-380 (1998)

10. Takahasi, S-E, Miura, T, Miyajima, S: On the Hyers-Ulam stability of the Banach space-valued differential equation $y^{\prime}=\lambda y$. Bull. Korean Math. Soc. 39, 309-315 (2002)

11. Miura, T, Jung, S-M, Takahasi, S-E: Hyers-Ulam-Rassias stability of the Banach space valued differential equations $y^{\prime}=\lambda y$. J. Korean Math. Soc. 41, 995-1005 (2004)

12. Jung, S-M: Legendre's differential equation and its Hyers-Ulam stability. Abstr. Appl. Anal. 2007, Article ID 56419 (2007)

13. Jung, S-M: Approximation of analytic functions by Airy functions. Integral Transforms Spec. Funct. 19(12), 885-891 (2008)

14. Jung, S-M: Approximation of analytic functions by Hermite functions. Bull. Sci. Math. 133, 756-764 (2009)

15. Jung, S-M: Approximation of analytic functions by Laguerre functions. Appl. Math. Comput. 218(3), 832-835 (2011)

16. Jung, S-M, Kim, B: Simple harmonic oscillator equation and its Hyers-Ulam stability. J. Funct. Spaces Appl. 2012, Article ID 382932 (2012)

17. Kim, B, Jung, S-M: Bessel's differential equation and its Hyers-Ulam stability. J. Inequal. Appl. 2007, Article ID 21640 (2007)

doi:10.1186/1029-242X-2013-3

Cite this article as: Jung: An approximation property of simple harmonic functions. Journal of Inequalities and Applications 2013 2013:3. 